International Journal of Motor Control and Learning (IJMCL)

\title{
The Transitional Effect of Fatigue Due to a Period of Physical and Mental Activity on the Level of Learning a Motor Skill
}

\author{
Mahin Mohammadpour ${ }^{a *}$, Mohammad Taghi Aghdasi $^{b}$ \\ ${ }^{a}$ M.S, Motor Behavior Department, Faculty of Sport Sciences, University of Tabriz, Tabriz, Iran \\ ${ }^{b}$ Professor, Motor Behavior Department, Faculty of Sport Sciences, University of Tabriz, Tabriz, Iran
}

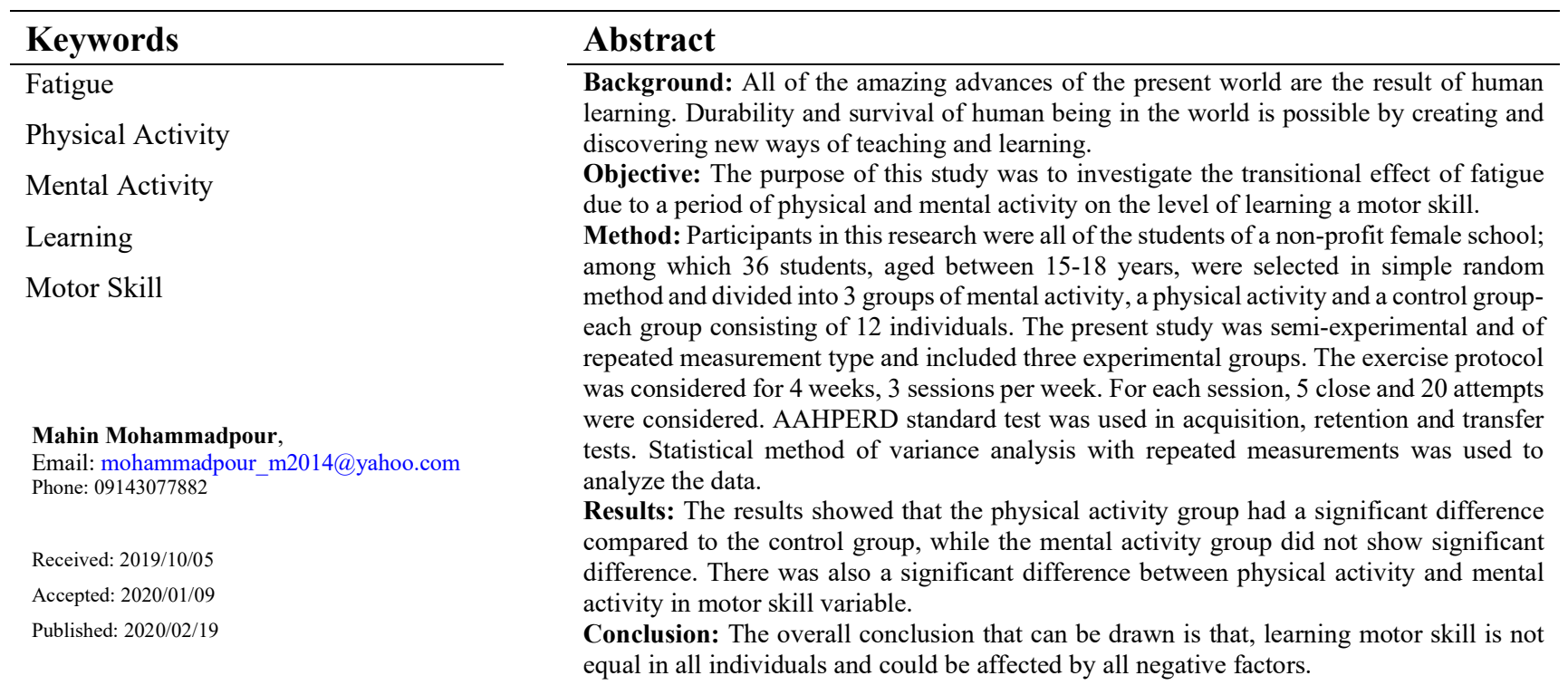

\section{Introduction}

Learning plays an important role in human life. The existence and survival of any culture depends on the ability of its members to learn a variety of skills, behavioral norms, beliefs and opinions. The survival of any living species requires that its members behave in such a way that they are compatible with the environment. This demand requires the ability to learn. Over a hundred years, many scholars have studied the factors affecting learning (Anderson, 2000). Performance and learning are the results of interaction among individual, assignment, and environment. Appropriate interaction of these three factors leads to better performance and learning. If the assignment requirements are not consistent with individual and environmental factors, performance will be weakened; so, individuals' adaptation to environmental conditions and assignment requirements may strengthen the engagement circle of learning factors (Sage, 1984). Some of the important factors in the effectiveness of exercise 
and learning that coaches should pay attention to are texture such as the characteristics of light, sound, spectators' noise and individual conditions such as fatigue caused by activity (mental or physical activity), stress due to competition conditions and the presence of rival team (Salehi \& et al, 2013).

Fatigue is a term used to describe the loss of physical function along with an increase in the actual or perceived difficulty of an assignment. From another point of view, fatigue refers to muscle weakness to maintain the required level of strength during exercise (Hatami \& et al, 2017). Physical activity and the fatigue caused by it, are among the limitations faced by the motor system of individuals (Gandevia, 2008).

Each skill, in addition to being involved in cognitive issues, must produce the least movement. In addition, each motor skill requires decision making that depends on cognitive knowledge (Schmidt, 1991). Although the beginning of the overall body fatigue is physiological, performing a mental activity and CNS fatigue provides the background for active muscles to reach the boundaries of their physiological abilities, so most of the athletes cut their workout or physical activity before their muscles are physiologically tired. (Big Land-Ritchie, 2013). Fatigue can be physical or mental. Mental fatigue is a kind of feeling of lack of power which, unlike muscle weakness, usually improves with rest (Arghami \& et al., 2013). Performing a mental activity and fatigue caused by it disrupt the mind and reduce agility as well willingness to do work, gradually or simultaneously (Halvani \& et al., 2007). Muscle fatigue is defined as a decrease in muscle's ability to produce optimal force after maximal physical activity, resulting in the discontinuation of the chain of events from the central nervous system to muscle fibers (Mcardle \& et al., 1998). It is believed that learning occurs as a result of physical activity in conditions of fatigue, this is referred to as reactive inhibition (Hoomanian \& et al., 2007; quoted by Abdoli et al., 2014). Although Granacer (2010) states that walking speed and step length of a singular skill (walk) and a dual skill (walk + cognitive) are not affected by fatigue; Masters (2008) reports that fatigue is an effective factor in learning a motor- throwing skill. In a general conclusion, Lynn Kerr, described fatigue as an effective variable on learning. Christina (2008) divided fatigue into two moderate and severe parts and stated that severe fatigue has an effect on learning and moderate fatigue affects performance. Rozand, Lebon \& Papaxanthis (2015) assessed the effect of mental fatigue on both-handed coordination, which showed that during mental fatigue, movements are performed slowly. In another study, these scholars showed that mental fatigue did not affect the performance of individuals in simple memory activities. These findings represent clear executive control during mental fatigue, which may be an explanation for common errors and inappropriate performance. They found that working on a heavy cognitive task for a long-time result in mental fatigue, which can reduce the efficiency of work (Linden \& et al., 2003). Further, Lorist \& et al., used EEG data to 
study the effect of working time on scheduling and selecting task. In their study, EEG data showed that by increasing working time, the extent of involvement of those areas of the brain associated with the implementation of executed control, decreases (frontal lobe). They also concluded that mental fatigue increases the number of errors and reaction time (quoted by Zoheiri \& et al., 2017). Amini and Samami (2017) in a study entitled "Comparing the Effect of Functional Fatigue on the Dynamic Balance of 18-48 years old Boys with Different Physical Types", studied the use of balance exercises before participating in any physical activity and appropriate exercises to improve the physical fitness of these individuals. Results showed that players in the penalty area have special skills and physical exhaustion, at this point, does not have a significant effect on the performance of players and does not reduce the quality of players performance at that point neither (Hatami et al., 2017). According to theories and researches carried out in this field, it can be stated that both types of mental and physical fatigue can affect performance and motor skills. Researches that have so far been on conducted fatigue have had little emphasis on the issue of motor learning and the effects of fatigue in different stages of learning and have not compared the mental and physical activity. The most important issue that matters here is the type of fatigue that affects the learning of a motor skill. Given that mental exhaustion affects the nervous system and neurons and also having in mind the fact that physical exertion affects the muscular system, the question: Do the effects of physical and mental activity and fatigue caused by them affect learning of a motor skill equallydespite different opinions in this case? Will these effects be preserved during the period of retention and transfer?

\section{Method}

\section{Research Method}

In this research, we used a semi-experimental method and three experimental groups which were used in order to examine the transitional effect of fatigue due to a period of physical and mental activity on the level of learning a motor skill by repeated measurement design with three groups (a group with doing mental activity, a group with physical activity and a control group). The process of categorizing and replacing individuals in these groups was beyond the knowledge of the participants.

\section{Participants and Statistical Sample of}

\section{Research}

The participants in the present study were all qualified students of Misag non- profit female school in Tabriz city, aged 16/44 $\pm 1 / 8$ years, of whom 36 students were selected in two stages (in the first stage, they were selected using a purposeful method, and in the second stage, they were selected randomly in order to replace them into groups). All of the subjects did not have any vision and musculoskeletal problems affecting research and were not familiar with the required skill. They were also right handed. It is worth noting that none of the participants had a history of 
basketball activity. After explaining the implementation of the test, the subjects were randomly divided into 3 groups: physical activity, mental and control.

\section{Data Collection Method}

The research was carried out in 4 weeks and 3 sessions per week. For each session, 5 close and 20 attempts were considered. In the first session, motor skill was taught to the subjects of all three groups, then, pre-test of motor skills was performed in all three groups and the registration of scores was done according to AAHPERD standard test (Average of 2 final attempts as a subject score). Then, in the next sessions, the two groups (physical activity and mental activity) performed a basketball dribble exercise after intervention, and one group performed it without intervention (control group). At the end of sixth session, the middle acquisition test and at the end of 12th session, the final acquisition test was carried out. The average of these two tests was recorded as the acquisition score of subjects. After 24 hours of the last session of exercise, the retention test was conducted and in order to implement the transfer test in motor skill (basketball dribbling), subjects were asked to perform this skill in the presence of active audiences.

\section{Research Tools}

It is preferred to use non-drug methods in order to intervene in the mental activity group and to create mental workload. To this end, subjects performed a relatively long-term mental activity.
Scholars such as Halvani \& et al (1386) and Boksem \& et al. (2005) indicated that a long-term concentration causes fatigue. So, in each session, after spending about 2 hours in class (mathematics, language) and without feeding in break time, and then 15 minutes' activity with Rubik's Cube, they were taught in the skill, according to the protocol prescribed in the Physical education class. VAS scale was used to measure mental fatigue of the 5 subjects (Arghami \& et al., 1392). VAS scale, is an ocular simulated scale which is considered as a self-report scale. What was used in this study was a simple ruler-like scale, with numbers from zero to ten centimeters ( 0 to $100 \mathrm{~mm})$ written on it. The subjects were asked to determine their mental fatigue trough this tool. In this scale, numbers equal to or less than $20 \mathrm{~mm}$ are reported as no pain, numbers greater than $20 \mathrm{~mm}$ to $40 \mathrm{~mm}$ as mild pain, numbers range from 40 to $70 \mathrm{~mm}$ as moderate pain, and numbers greater than 70 as severe pain.

In order to intervene in the physical activity group, in each session, subjects performed the Bruce aerobic exercise test (Sadegh Afrozeh et al, 1393) before starting classes and in the physical education class. The purpose of the Bruce treadmill test was to estimate the aerobic capacity of individuals with low mobility, active people and athletics. This test has a 10-step protocol, starting at $74.7 \mathrm{~km} / \mathrm{h}$ and a slope of $10 \%$; the percentage of slope as well the speed of the treadmill increases according to the standard table of the Bruce test. The test lasts as long as the subject is tired and unable to continue the test. The test time is measured in minutes and up to two decimal places 
from the starting point until the person is unable to continue the activity. It is used in a fraction of a minute in estimating equations in order to estimate the aerobic capacity of active and sedentary men and women. Given that, only the subjects' fatigue was considered in this study, the time was recorded until athletes were exhausted. This was done because a competition exists between subjects in terms of the maximum time to get exhausted (Sadegh Afrozeh \& et, al., 1393).

The RPE perceived pressure and hardness scale was used to measure the amount of physical fatigue. It is a 15-degree class scale for rating perceived pressure and is used as a training intensity guide. In this way, individuals express their understanding of the difficulty of a work they are doing. The RPE ratings are used to determine when a person achieves his/her maximum tolerance. It is known that this is the most appropriate method for choosing the right intensity of exercise and can be used to complete estimations of heartbeat. It has been shown that the RPE ratio scale is highly correlated with the lactic acid in the blood and the amount of muscle lactic acid, both of which are biochemical symptoms of cardiac respiratory and muscular fatigue (Borg, 1982). Borg also announced that the scale would be appropriate $=0.92$.

AAHPERD standard test was used in acquisition, retention, and transfer tests in order to measure the performance of subjects. This test is done 3 times. The first test has a training aspect and the next two are recorded. The subject stands with a ball next to the cone $\mathrm{A}$ and the non-superior hand becomes a dribbler. With the present and go sign, the subject dribbles with non-superior hand and goes to the cone $\mathrm{B}$, rounds out the cone $\mathrm{B}$, and then continues the path with superior hand and changes her/his hands with respect to the appropriate position. If the ball is lost, the subject will regain the ball and will continue from where the ball's control is lost. If the offense (Running and Double) happens, or if the subject or ball goes out of the path or the subject is tired, the test stops, the test will continue on the point where the ball's control is lost. The score for each test is the elapsed time that the path is legally completed. The timer starts with the "Go" sign and stops when both feet cross the finish line. The time for each test is recorded around $0.1 \mathrm{sec}$.

\section{Results}

The participants in this study included three experimental groups: (physical activity) (mental activity) and control. In this section, the characteristics of the subjects such as height, weight, age and body mass index are presented. Table 4-1 presents mean and standard deviation of the individual characteristics of the subjects in these groups. 


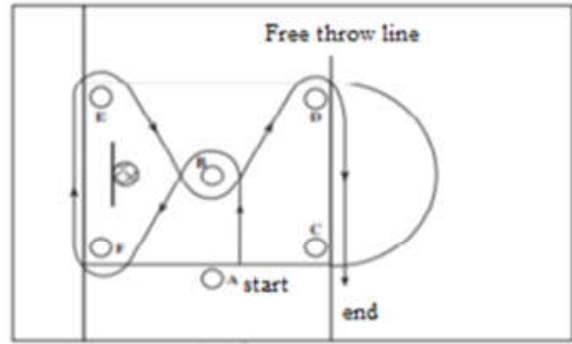

Dribble starts with right hand (in left-handed subjects)

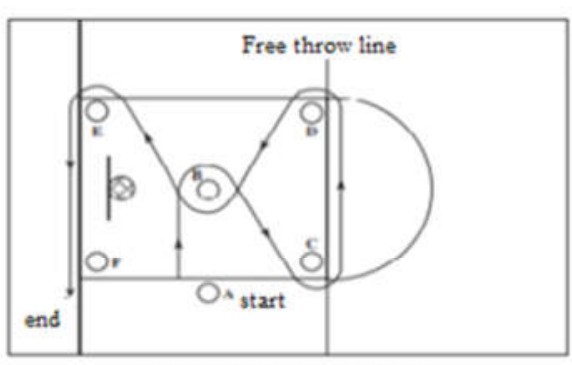

Dribble starts with left hand (in right-handed subjects)

Figure 1. How to Run AAHPERD Basketball Control Dribble Test.

\section{Information analysis method}

Analysis of variance method with repeated measurements was used to examine the significant differences between and within groups. In order to be sure in all statistical works, the alpha level is considered $\mathrm{P} \leq 0.05$. SPSS version 21 was used to analysis the data.

Table1. Individual Characteristics of Subjects.

\begin{tabular}{ccccc}
\hline \multirow{2}{*}{ Group } & Age (year) & Height $(\mathrm{cm})$ & Weight & Body Mass Index \\
\cline { 2 - 5 } & SD \pm Mean & SD \pm Mean & SD \pm Mean & SD \pm Mean \\
\hline Physical Activity & $16.17 \pm 1.11$ & $163.08 \pm 5.77$ & $67.71 \pm 6.37$ & $25.47 \pm 2.27$ \\
\hline Mental Activity & $16.67 \pm 1.23$ & $162.58 \pm 4.18$ & $65.75 \pm 5.51$ & $24.91 \pm 2.37$ \\
\hline Control & $16.50 \pm 1.24$ & $164.67 \pm 4.47$ & $66.58 \pm 5.72$ & $24.97 \pm 2.48$ \\
\hline SD, standard deviation. & & & &
\end{tabular}

Table2. Mean and Standard Deviation of Motor Skill Variable for All of Groups.

\begin{tabular}{cccccc}
\hline Variable & Groups & Pre-test & Acquisition & Retention & Transfer \\
\hline \multirow{4}{*}{ Motor skill } & $\begin{array}{c}\text { Physical } \\
\text { Activity }\end{array}$ & $26.22 \pm 4.22$ & $26.10 \pm 4.47$ & $25.90 \pm 3.80$ & $25.77 \pm 4.02$ \\
\cline { 2 - 6 } & \begin{tabular}{c} 
Mental Activity \\
\cline { 2 - 6 }
\end{tabular} & $27.05 \pm 3.69$ & $23.28 \pm 3.59$ & $20.64 \pm 3.20$ & $16.99 \pm 3.66$ \\
\cline { 2 - 6 } & Control & $26.68 \pm 5.57$ & $21.36 \pm 2.85$ & $19.98 \pm 3.11$ & $18.40 \pm 3.90$ \\
\hline
\end{tabular}

\section{The Descriptive Findings of Mental and Physical Fatigue Indexes Based on VAS and Borg Scale}

Table 3. Descriptive Statistics of Mental and Physical Fatigue Indexes.

\begin{tabular}{cccc}
\hline \multirow{2}{*}{ variables } & groups & stage & SD mean \\
\hline \multirow{3}{*}{ Motor skill } & \multirow{3}{*}{ Mental activity } & Before Class & $25.86 \pm 7.48$ \\
\cline { 2 - 4 } & & After Class & $39.55 \pm 9.68$ \\
\cline { 2 - 4 } & & After Solving & $21.78 \pm 5.87$ \\
\cline { 2 - 4 } & \multirow{2}{*}{ Physical activity } & Rubik's Cube & $11.56 \pm 2.17$ \\
\cline { 2 - 4 } & & Before Bruce Test & $17.59 \pm 0.81$ \\
\hline
\end{tabular}

Based on the descriptive statistics, mental and physical fatigue indexes after intervention have increased, compared to the pre-test. 
Table 4. Results of Analysis of Variance with Repeated Measurements for Intra-group Differences in Physical Activity Group.

\begin{tabular}{cccccc} 
Source & \multicolumn{2}{c}{ Type III Sum of Squares } & Df & Mean Square & F \\
\hline Intercept & 1.510 & 3 & 0.503 & 1.676 & 0.191 \\
\hline Error & 9.915 & 33 & 0.300 & & \\
\hline
\end{tabular}

The results of intra-group comparisons showed that four weeks of physical activity intervention $(\mathrm{p}=$
0.197) caused a significant difference in motor skills compared to the untested stage.

Table 5. Results of Analysis of Variance with Repeated Measurements for Intra-group Differences in Mental Activity Group.

\begin{tabular}{cccccc} 
Source & Type III Sum of Squares & df & Mean Square & F & t \\
& & & & & \\
& 648.82 & 3 & 216.276 & 42.653 & 0.0001 \\
\hline Intercept & 167.332 & 33 & 5.071 & & \\
\hline Error & & 33 &
\end{tabular}

The results of intra-group comparisons showed that the four-week intervention of mental activity $(\mathrm{p}=$
0.0001) caused a significant difference in motor skills compared to the pre-test stage.

Table 6. Significant Comparison between Different Stages in Mental Activity Group.

\begin{tabular}{ccccc}
\hline \multirow{2}{*}{ Group } & Stages & Mean Difference & $\begin{array}{c}\text { Error Deviation from } \\
\text { mean }\end{array}$ & Significance \\
\hline \multirow{3}{*}{ Mental Activity } & Pretest- Acquisition & 3.764 & 1.031 & 0.023 \\
\cline { 2 - 5 } & Pretest - Retention & 6.406 & 1.108 & 0.001 \\
\cline { 2 - 5 } & Pretest - Transfer & 10.058 & 1.319 & 0.0001 \\
\cline { 2 - 5 } & Acquisition- Retention & 2.642 & 0.538 & 0.003 \\
\cline { 2 - 5 } & Acquisition- Transfer & 6.293 & 0.677 & 0.0001 \\
\cline { 2 - 5 } & Retention- Transfer & 3.652 & 0.541 & 0.0001 \\
\hline
\end{tabular}

Table 7. Results of Analysis of Variance with Repeated Measurements to Compare the Groups in Motor Skill Variable.

\begin{tabular}{cccc}
\hline Groups & Mean Difference & $\begin{array}{c}\text { Error Deviation from } \\
\text { Mean }\end{array}$ & Significance \\
\hline $\begin{array}{c}\text { Physical Activity- } \\
\text { Mental Activity }\end{array}$ & $4 / 009$ & $1 / 474$ & $0 / 031$ \\
\hline $\begin{array}{c}\text { Physical Activity- } \\
\text { Control }\end{array}$ & $4 / 392$ & $1 / 474$ & $0 / 016$ \\
\hline $\begin{array}{c}\text { Mental Activity- } \\
\text { Control }\end{array}$ & $0 / 383$ & $1 / 474$ & $0 / 999$ \\
\hline
\end{tabular}

The results showed a significant difference in physical activity compared to the control group $(\mathrm{P}=0.016)$, while mental activity did not have a significance difference $(\mathrm{P}=0.999)$. Also, there is a significant difference between the two groups of mental and physical activity $(\mathrm{P}=0.031)$ in motor skill variable (table 4-6). So, the first assumption that the effect of a period of physical and mental activity on the acquisition, retention and transfer of motor skills is not different is rejected and the hypothesis of this research is confirmed. 
Table 8. ANOVA Test Results to Compare the Stages between Groups in Motor Skill.

\begin{tabular}{ccccc}
\hline \multirow{2}{*}{ Groups } & Stages & Mean Difference & $\begin{array}{c}\text { Error Deviation } \\
\text { from Mean }\end{array}$ & Significance \\
\hline \multirow{3}{*}{$\begin{array}{c}\text { Physical Activity- } \\
\text { Mental Activity }\end{array}$} & Pre-test & 0.821 & 1.864 & 0.899 \\
\cline { 2 - 5 } & Acquisition & 2.822 & 1.51 & 0.164 \\
\cline { 2 - 4 } & Retention & 5.258 & 1.384 & 0.002 \\
\hline \multirow{2}{*}{$\begin{array}{c}\text { Transfer } \\
\text { Physical Activity- } \\
\text { Control }\end{array}$} & Pre-test & 8.778 & 1.579 & 0.0001 \\
\cline { 2 - 4 } & Acquisition & 0.457 & 1.864 & 0.698 \\
\cline { 2 - 4 } & Retention & 4.745 & 1.51 & 0.010 \\
\hline
\end{tabular}

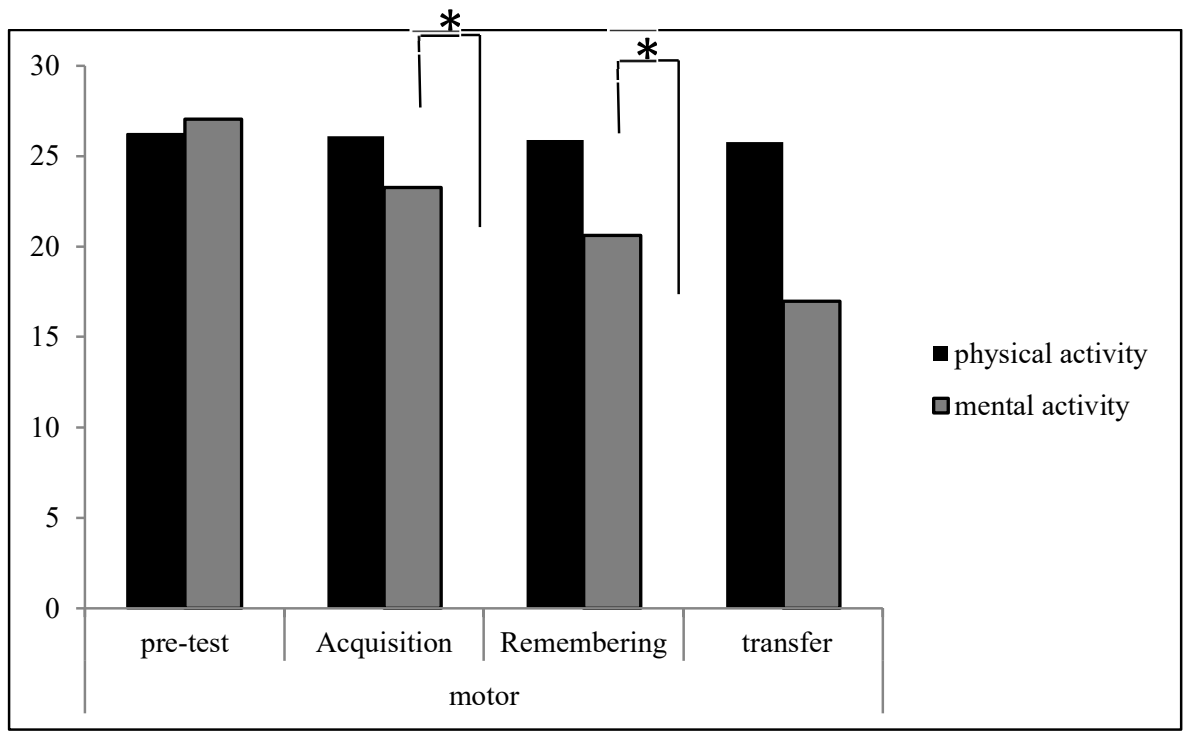

Chart 1. Changes between Motor Skill Stages in Two Physical and Mental Activity Groups.

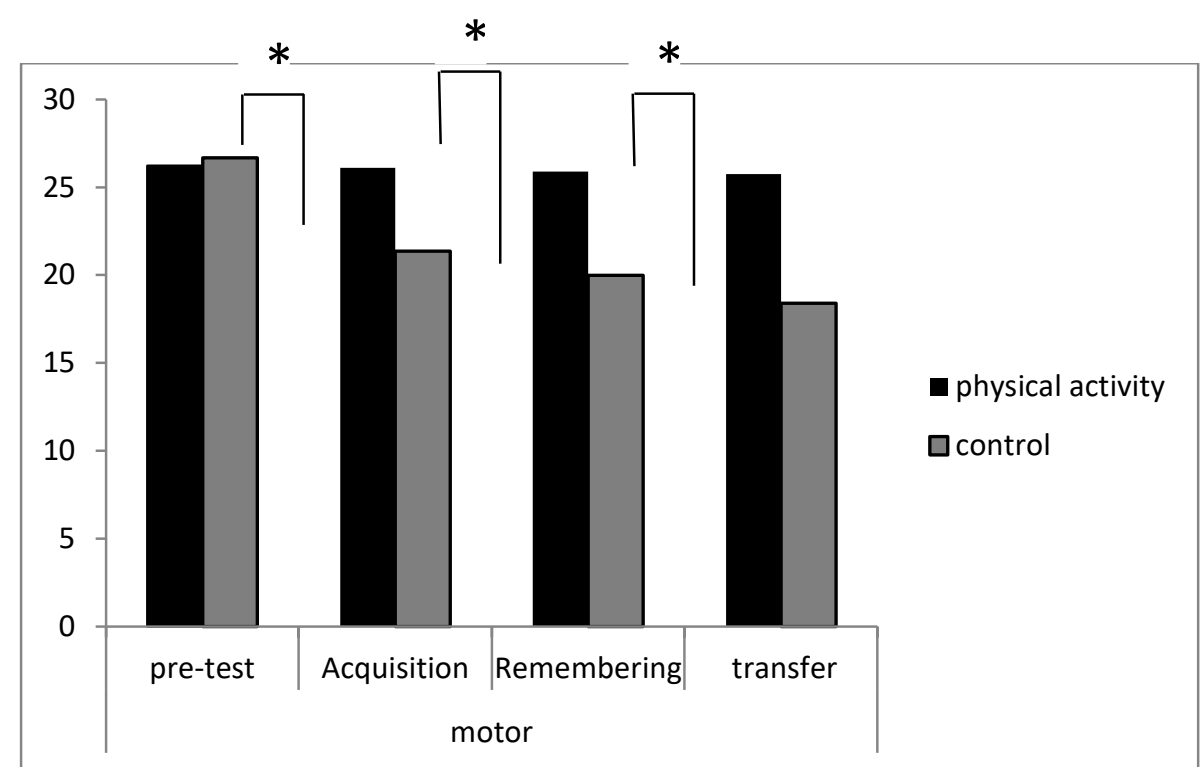

Chart 2. Changes between motor skill stages in two physical activity and control groups. 


\section{Discussion and Conclusion}

One of the important aspects in individuals' life is learning motor behavior. The study of motor learning is significantly different from the performance study. In the performance study, emphasis is on the changes resulting from the direct result of exercise (Hemayat Talab \& et al., 2009). The most important subjects in learning motor skills are training and exercise conditions. Experts have always tried to examine the factors affecting learning and the performance of motor skills. Since the most important factor is learning of intentional exercises, therefore, a coach or teacher's training plan should be complete and comprehensive, given the nature of the skill and the other influential factors and also must reflect the conditions in which skills are performed (Magil, 1380). Useful learning leads to time saving, energy-saving of learners, and effective guidance in order to achieve the most important goals of sport plans such as training a good competitive athlete and gaining pride. Therefore, use of all principles of motor learning in teaching and gaining sport and motor skills are necessary to create new motor behavior (Nabilpour \& Aghababa, 1391). In this context, coaches must take actions and design training environments in such a way that maximizes learner's performance. Some of the important factors in the effectiveness of exercise and learning that coaches should pay attention to them are texture such as the characteristics of light, sound, spectator's noise and individual conditions such as fatigue caused by activity (mental or physical activity), stress due to competition conditions and the presence of rival team (Salehi \& et al, 1392).

Although the beginning of the overall body fatigue is physiological, performing a mental activity and CNS fatigue provides the background for active muscles to reach the boundaries of their physiological abilities. Because of the fact that most of the athletes cut their workout or physical activity before their muscles are physiologically tired, they can be encouraged to continue their work (Big Land-Ritchie, 2013). Fatigue can lead to a disorder in coordination, loss of motion elegance, inaccuracy, and reduced focus and increased response time for individuals as well as changes in motor commands (Hatami \& et al., 2017). Some studies have shown that physical stress has a positive effect on the cognitive performance of individuals, while others have achieved contradictory results and considered this work to be harmful or neutral. In fact, it is difficult to compare the results of such studies, because the intensity and duration of exercise and the type of cognitive tests used vary from one to another (Covassin \& el al., 2007). The results of this study showed that, Interventions were carried out (physical activity and mental activity) have different effect on learning motor skill. In other words, the intervention of physical activity disturbes the learning process of motor skills. Fatigue, due to physical activity, may prevent subjects compared to pre-test, while mental activity intervention did not disturb the learning process of motor skills; in other words, the level of performance in acquisition, retention and transfer stages in the 
physical activity group in motor skill was lower than the mental activity group and the control group. The first part of the findings of the destructive effect of fatigue due to physical activity on learning motor skills was similar to the results of Cignetti \& et al (2004), but it differs from the results of Tanaka et al. (2015). The second part of the findings, which showed that the effect of fatigue due to mental activity on the level of motor skills learning was similar to those of Linden, Michelle \& Meijman (2002); and differs from the results of Mun, Mark \& Kim (2014).

In explaining these findings, it can be stated that motor skill depends on the performance of muscles and organ. Fatigue due to physical activity, is defined as decrease in the ability of muscles to produce the desired power that occurs because of the discontinuation of the chain of events from the central nervous system to muscle fibers (Mcardle $\&$ et al., 1998). So, this type of fatigue interferes with the learning of motor skills and mental fatigue which is a kind of lack of power. It is unlike muscle weakness that usually improves with rest and does not disrupt the learning process of motor skills (Arghami \& et al, 1392).

It can be stated that human behavior varies depending on the circumstances in which learning occurs, and considering that motor skill is related to the performance of muscles and organs, the physical or mental activity and the resulting fatigue have different effects on learning of these skills.

In a general conclusion of the results, we can say that learning motor skills is not equal in all people and can be influenced by individual and environmental factors. Meanwhile, it is necessary to pay attention to these factors, in order to choose the correct method of teaching and learning. Accordingly, it is recommended that coaches should identify and control those factors disturbing learning skills before training.

\section{References}

1. Abdoli, Behrouz. Farsi, Alireza. Teymouri Khoravi, Mostafa. Spring and summer (2014). " Comparing the effect of mental exercise and physical exercise in fatigue and non-fatigue condition on learning the generalized motion program and parameter". growth and mental learning journal, volume 19. p.p: 25-38.

2. Amini, hope. Shemami, Nader Summer (2017). "Comparison of the Effect of Functional Fatigue on the Dynamic Balance of 18-25 Years Boys with Different Physical Types". Journal of Tabari Prevention Medicine. Second course Second Issue. P. 35-26.

3. Anderson J R. (2000). Learning and memory: An integrated approach. 2nd Ed. New York: Wiley. p. 1-5.

4. Arghami, Shirazeh. Ghoreyshi, Abolfazl. Kamali, Kurosh. And Farhadi, Masood (2013). " The study of the consistency of the results of measurement of mental fatigue with the Visual Analog Scale (VAS) and device". Ergonomics magazine. No. 1, p.p: 66-72.

5. Big land-Ritchie, B. (1987). Central and peripheral fatigue in sustained maximum Voluntary contractions of human quadriceps muscle. Clin Sci Mol Med.54(6):p. 609-614.

6. Boksem MS, Meijman T, and Lorist M. (2005). Effects of mental fatigue on attention: An ERP study. Cogn Brain Res. 25:107-116.

7. Borg GAV. (1982). Psychophysical bases of perceived exertion. Med Sci Sport Exerc.14: 377-381.

8. Christina, Robert (2008). " Sport Skills Training, Coach Guide". Translated by Mohammad Taghi Aghdasi. Tabriz University publications.

9. Cignetti F, Schena F, Rouard A. (2009). Effects of fatigue on inter-cycle variability in crosscountry skiing. Biomech.42:1452-9.

10. Gandevia S C.(2088). Spinal and supraspinal factors in human muscle fatigue. Gait Posture.; 11: 21-37.

11. Granacher Urs, Wolf Irene, Wehrle Anja, Bridenbaugh Stephanie; Kressig Reto W; ( 2010). Effects of muscle fatigue on gait characteristics under single and dual-task conditions in young and older adults, Journal of NeuroEngineering and Rehabilitation, 7:56.

12. Halvani Gh, Baghiani moghadam M H, Rezaei MH. (2007). Fatigue situation in tile industries workers. Iran Occupational Health Journal. 4:57-63.

13. Hatami, Farzaneh. Tahmasebi, Farshid. Mirmiran, Elaheh (2017). " The Effect of Physical Fatigue on basketball Special Shooting Skill in expert players" Growth and Learning journal, No 3. p.p: 493-513. 
14. Hemayattalab, Rasoul. Moradi, Ali. Borumand, Mohammadreza. And Fakhrpour, Roghayeh (2009). " The effect of interference on the acquisition, retention and transfer of volleyball service skills", growth and motor learning journal, No 3, p.p: 5-22.

15. Humaneyan, D., Sheykh, M., Bagherzade, F., khosravi, N., Kalkhoran, J, F. (2007). "The effect of various method of mental practice on chained reaction time", Harkat, 34.

16. Lorist, Monicque M., Merel Klein, Sander Nieuwenhuis, Ritske Jong, Gijsbertus Mulder, and Theo F. Meijman.(200•). "Mental fatigue and task control: planning and preparation." Psychophysiology 37, no.614-625.

17. Lynn Kerr Tracey.(1998). an analysis of the effects of fatigue and specificity on motor learning, thesis master of science in the school of kinesiology Simon Fraser University.

18. Magil, Richard. E. (2001). " Motor Learning - Concepts and Applications". Translated by Seyed Mohammad Kazem Vaez Mousavi, Masoumeh Shojaei, Tehran, Hananeh publications. First edition.

19. Masters RSW, pool ton JM, Maxwell JP. ( 2008). Stable implicit motor processes despite aerobic loco motor fatigue; Consciousness and Cognition. San Diego. Vol. 17, Iss. 1; pg..335.

20. Mcardle W, Katch F, Katch V. (1998). Exercise physiology; 4 thedition. US: Lippincott Williams \& Wilkins: New Work.400-2.

21. Mun, S., E.-S. Kim, and M.-C. Park.(2014)." Effect of mental fatigue caused by mobile $3 \mathrm{D}$ viewing on selective attention: An ERP study." International Journal of Psychophysiology, 94(3): p. 373-381.

22. Rozand V, Lebon F, Papaxanthis C, Lepers R. (2015)." Effect of mental fatigue on speed-accuracy trade-off. Neuroscience ". 297, p.219-230.

23. Sadegh afrouzeh, Mohammad. Mohammadzadeh Jahatlu, Hasan. Edrisi Kolur, Mostafa. Afrozeh, Ali (2014). "the impact of obvious and implicit motivational self-talk on the level of perceived pressure and performance in exercise to exhaustion in wrestlers", growth and mental learning journal, No 2. p.p: 245-257.

24. Sage Gh. (1984). Motor learning and control: A neuropsychological approach. 1st Ed. Dubuque: Brown. P. 18-32.

25. Salehi, Hamid. Movahedi, Ahmadreza. Moradi, Jalil (2013). " Does the practice in special sight conditions improve the accuracy of Free Basketball Throwing Skill?" growth and mental learning journal, No 11. p.p: 23-38.

26. Schmidt, R A. (1986). Toward a better understanding of the acquisition of skill: theoretical and practical contributions of the task approach: DTIC Document...

27. Tanaka M, Ishii A, Watanabe Y. (2015). Physical fatigue increases neural activation during eyesclosed state: a magnetoencephalography study. Behavioral and Brain Functions. 11(1):35.

28. Van der Linden, D., M. Frese, and T.F. Meijman. (2003). "Mental fatigue and the control of cognitive processes: effects on perseveration and planning". Acta Psychologic. 113(1): p. 45-65.
29. Zoheyri, Ali. Shahbazi, Mahdi. Kordi, Mohammadreza. Fazal Kolkhuran, Jamal. Spring (2017). " The Effect of Central and Environmental Fatigue on the Coordination of Academic Athletes". Growth and motor-sports learning research. Volume 9, No 1. p.p: 123- 136. 